\title{
Quantitative Determination of the Metastability of Flat Ag Overlayers on GaAs(110)
}

\author{
Hongbin Yu, ${ }^{1}$ C. S. Jiang, ${ }^{1}$ Ph. Ebert, ${ }^{2}$ X. D. Wang, ${ }^{1}$ J. M. White,,${ }^{3,45}$ Qian Niu, ${ }^{1,5}$ Zhenyu Zhang, ${ }^{6}$ and C. K. Shih ${ }^{1,4,5}$ \\ ${ }^{1}$ Department of Physics, The University of Texas at Austin, Austin, Texas 78712 \\ ${ }^{2}$ Institut für Festkörperforschung, Forschungszentrum Jülich GmbH, 52425 Jülich, Germany \\ ${ }^{3}$ Department of Chemistry and Bio-Chemistry, The University of Texas at Austin, Austin, Texas 78712 \\ ${ }^{4}$ Texas Materials Institute, The University of Texas at Austin, Austin, Texas 78712 \\ ${ }^{5}$ Center for Nano and Molecular Science and Technology, The University of Texas at Austin, Austin, Texas 78712 \\ ${ }^{6}$ Solid State Division, Oak Ridge National Laboratory, Oak Ridge, Tennessee 37831-6032
}

(Received 22 December 2000; published 14 December 2001)

Atomically flat ultrathin $\mathrm{Ag}$ films on $\mathrm{GaAs}(110)$ can be formed through a kinetic pathway. However, such films are metastable and will transform to 3D islands upon high temperature annealing. Using scanning tunneling microscopy, we have measured quantitatively the layer-resolved metastability of flat Ag overlayers as they evolve toward their stable state, and deduced the corresponding kinetic barrier the system has to overcome in reaching the stable state. These results indicate that the metastability of the Ag overlayer is defined by the quantum nature of the conduction electrons confined within the overlayer.

DOI: 10.1103/PhysRevLett.88.016102

In today's search for novel materials, metastable configurations of complex systems [1] are attracting much interest, because they often possess intriguing properties that may lead to innovative technological advancements. However, as a prerequisite for any potential device application, we must explore the degree of metastability of such materials, namely, how far they are from their thermodynamically stable configuration. In particular, it is desirable to know the energy barrier preventing a specific metastable configuration of a given macroscopic system from reaching its thermodynamically stable state, and to identify the dominant physical factors defining the metastable state.

One example of such a metastable system is Ag overlayers on a GaAs(110) surface, which can be grown atomically flat using a two-step procedure [2-4]. This particular growth procedure (low-temperature deposition followed by thermal annealing) allows the film to follow a special kinetic pathway and reach a fully wetting and atomically flat two-dimensional morphology, although in thermal equilibrium the $\mathrm{Ag}-\mathrm{GaAs}$ system is nonwetting and minimizes the interface area by forming three-dimensional mounds [5].

In this Letter we determine quantitatively the energetics that govern the wetting-nonwetting transition of the $\mathrm{Ag}$ overlayers grown on GaAs(110) as well as the underlying physical factors defining the metastability of the flat films. More specifically, we measure the layer-resolved relative energies of the flat overlayer in different metastable configurations, and deduce the kinetic barrier the initial system must overcome in order to transform from the wetting to the nonwetting morphology. Finally, a comparison with existing theoretical models indicates that the metastable configuration of the Ag overlayer is defined by the confined motion of the conduction electrons within the overlayer.

The experiments were performed in an ultrahigh vacuum system with a base pressure of $3 \times 10^{-9} \mathrm{~Pa}$. The Ag films were deposited on in situ cleaved GaAs(110) sur-
PACS numbers: 68.55.Jk, 68.35.Ct, 68.37.Ef, 73.21.Fg

faces at temperatures below $140 \mathrm{~K}$, followed by annealing to room temperature. The thickness of the film is monitored by a calibrated quartz crystal. At this stage we have to recall some important features: First, for all the film thicknesses investigated in this work, the as-deposited film at low temperature $(<140 \mathrm{~K})$ consists of nanoclusters of a few nanometers in diameter. Upon annealing to room temperature, the Ag clusters start to coalesce at about $225 \mathrm{~K}$, forming small flat terraces, and eventually evolving into a flat film at room temperature. This is a kinetic pathway leading to the metastable flat film morphology. Second, previous scanning tunneling microscopy (STM) studies revealed that the flat film morphology can develop only in a fully wetting layer for a thickness of more than 6 monolayers (ML) $[2,6]$. Below that threshold or critical thickness, the film consists of islands of $6 \mathrm{ML}$ in height surrounded by the bare $\mathrm{GaAs}(110)$ substrate [becoming voids exposing the GaAs(110) substrate if the $6 \mathrm{ML}$ high Ag islands are interconnected]. Once the fully wetting two-dimensional film has been reached, annealing to a higher temperature, e.g., $670 \mathrm{~K}$, leads to the irreversible transformation of the film to a collection of isolated mounds, or 3D islands [7]. In order to explore this metastability and the barriers involved for this transition with atomic-layer resolution, the films were deposited with thicknesses close to the critical value of $6 \mathrm{ML}$, and the time-dependent evolution of the film morphology was monitored using STM.

Figure 1 shows the morphological evolution of the Ag-film deposited at a thickness of $6.4 \mathrm{ML}$, i.e., close to the critical thickness of $6 \mathrm{ML}$. After warming up the substrate to $300 \mathrm{~K}$ within $10 \mathrm{~min}$, the film first exhibits local regions of flat films with 6 and 7 ML thicknesses, mixed with certain concentration of voids, which expose the GaAs substrate (Fig. 1a). In the early stages at room temperature, the film is driven toward the formation of flat film of a single thickness of $7 \mathrm{ML}$ associated with an enlargement of voids as required by mass conservation 

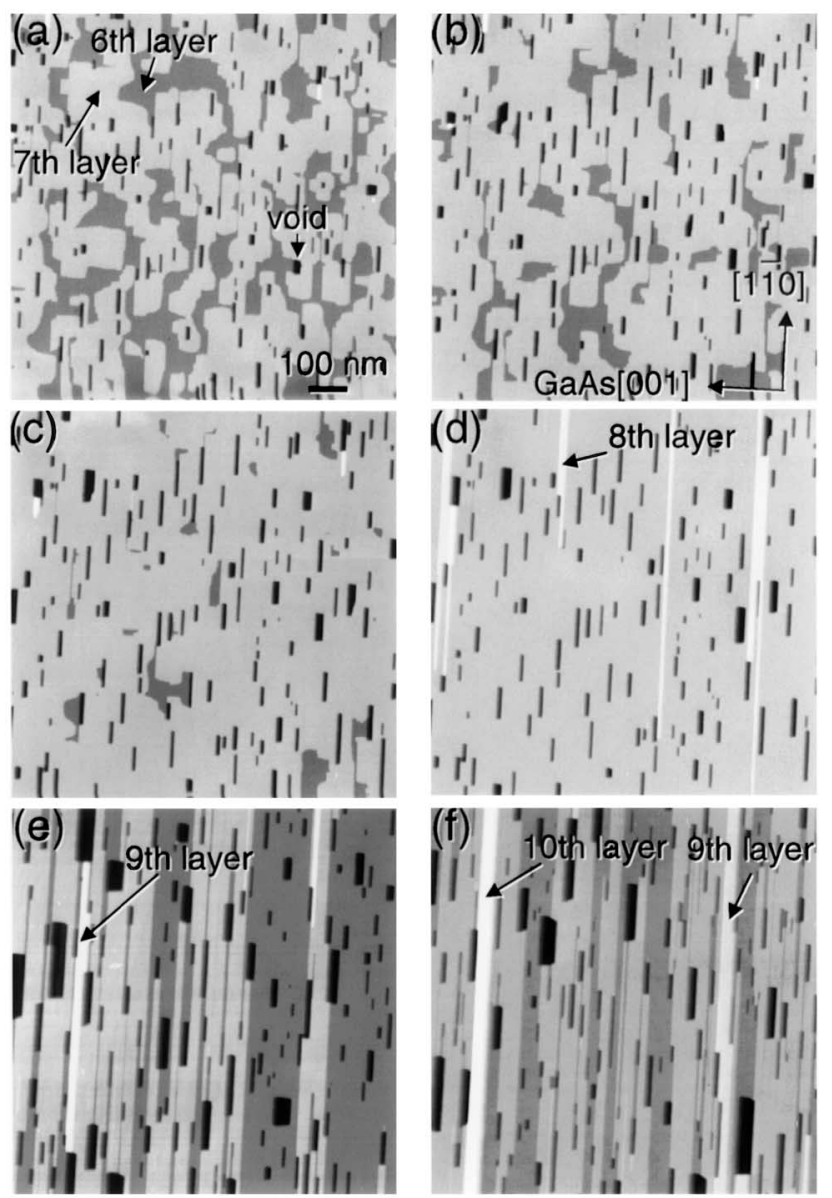

FIG. 1. STM images taken (a) 162, (b) 292, (c) 422, (d) 1387, (e) 4577 , and (f) $6363 \mathrm{~min}$ after warm-up to room temperature. Images (a) to (c) show the completion of the 7th layer by transferring Ag atoms from the voids. In image (d), the 8th layer starts to grow on top of the 7th layer, and images (e) and (f) show the formation of the 9th and 10th layers, respectively.

(Figs. 1a-1c). Note that the observation of a local $N$ th layer corresponds to a film with $N$ monolayer local thickness. The film continues to evolve further at room temperature and develop a multilayer morphology with consecutive emergence of the 8th, 9th, and even 10th layer (Figs. 1d-1f) although the deposition is stopped. The evolution shown here is the initial stage of the transition to a three-dimensional mound structure slowed only by the rather low kinetic rate at room temperature. We note that during the evolution to a multilayer film, the islands of a new layer are initiated at the edges of the voids and have elongated (needlelike) shapes.

A completely different behavior is found for $\mathrm{Ag}$ films prepared without voids, e.g., by depositing Ag with a thickness much higher than $6 \mathrm{ML}$. Figure 2 shows the evolution of a $25 \mathrm{ML} \mathrm{Ag} \mathrm{film.} \mathrm{After} \mathrm{rapid} \mathrm{warm-up,} \mathrm{the} \mathrm{film}$ initially exhibits a multilayer morphology (exposing in this case five layers) free of voids (Fig. 2a). This void-free film evolves toward a smooth surface. The number of layers decreases from five in Fig. 2a to three in Fig. 3b, and upon annealing further to almost two in Fig. 2c, close to the ultimate flat film morphology.
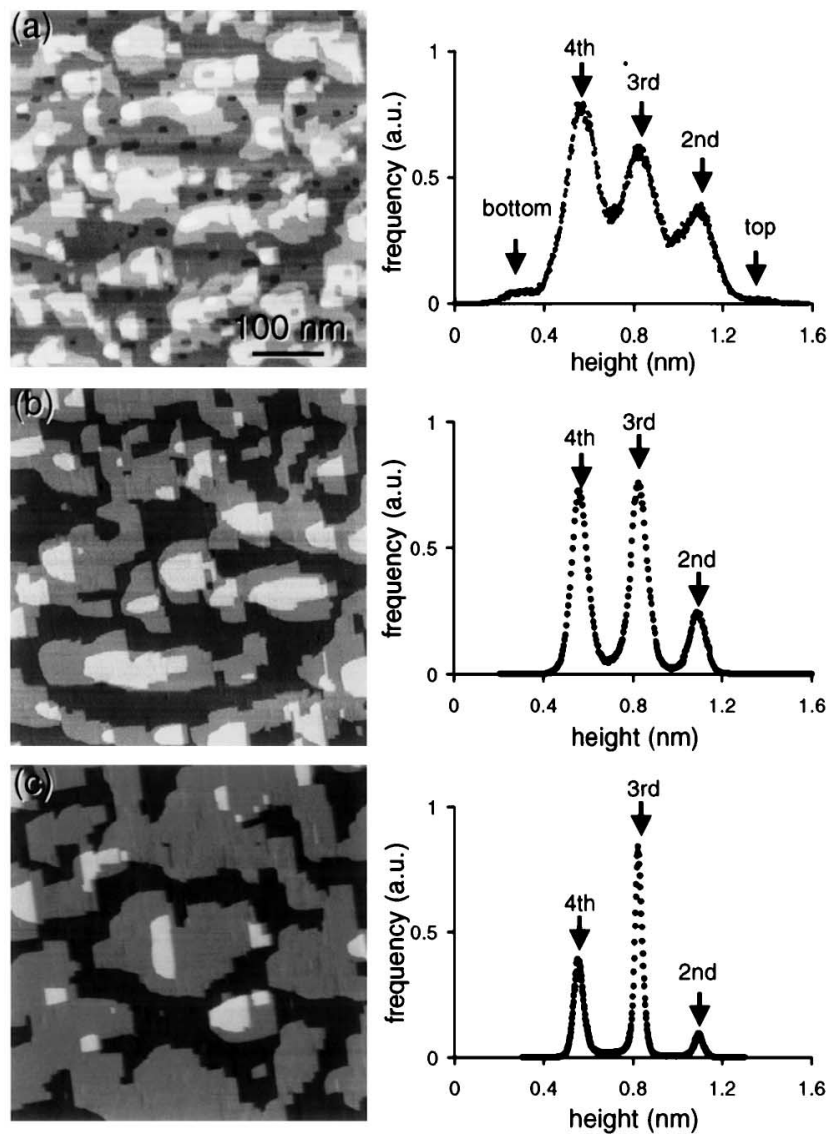

FIG. 2. STM images (left) and the respective height distributions (right) of a $25 \mathrm{ML}$ thick film. (a) and (b) show that at room temperature the initially exposed five layers of the film evolve after $13 \mathrm{~h}$ into a morphology with three exposed layers. (c) is obtained after annealing the film to $385 \mathrm{~K}$ for $20 \mathrm{~min}$. A further evolution toward a film with two exposed layers is visible.

These two examples exhibit morphological evolutions with opposite directions, i.e., roughening connected with a formation of mounds in films with voids, and smoothening of rough films without voids. These observations suggest that the voids act as seeds for the transition toward the nonwetting three-dimensional equilibrium film morphology. This interpretation is corroborated by the fact that $\mathrm{Ag}$ atoms are always transferred from void edges onto the

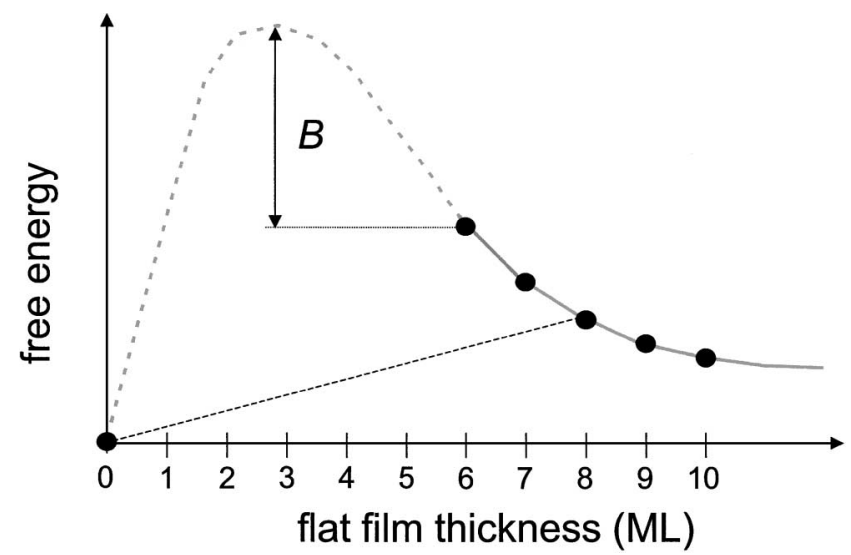

FIG. 3. Schematic diagram of free energy as a function of the film thickness used for the interpretation of the data. 
surface and that with no voids no roughening occurs unless upon very high-temperature annealing. Note that the void density depends sensitively on the initial deposition conditions and the warming up rate from low to room temperature (in this case $10 \mathrm{~min}$ ). A fast warm-up yields either voids for thin films or rough surfaces for thicker films. A slow warm-up produces nearly perfect flat $\mathrm{Ag}$ layer for all thicknesses above $6 \mathrm{ML}$. We also found that once the flat film is formed, the void density remained essentially constant during the evolution toward the multilayer morphology. Finally, the creation of islands on top of the film implies an "uphill" motion of atoms [6], and their needlelike shape indicates a diffusion anisotropy [8]. The latter is conceivable due to the pronounced anisotropy of the superstructure leading to stripes [4].

The general behavior observed here allows us to explore the layer-dependent thermodynamic stability and the relative value of the free energy as a function of the layer thickness. Figure 3 shows a schematic of the layerdependent free-energy diagram $F(N)$ versus the number of layers $N$, suggested by our experiment. In the absence of voids, the system is locally stable against phase separation into regions of lower and higher thicknesses (Fig. 2). Thus $F(N)$ must lie below the line connecting $F(N-1)$ and $F(N+1)$. Consequently, the free-energy plot as a function of layer thickness must have an upward curvature for $N \geq N_{\mathrm{C}}$, with $N_{\mathrm{C}}$ being the critical thickness. On the other hand, in the presence of voids, the system is globally unstable against phase separation into regions of voids $(N=0)$ and higher thicknesses. Therefore, $F(N)$ lies above the line that connects $F(0)$ with $F(N+1)$ for $N \geq N_{\mathrm{C}}$ (see dotted line in Fig. 3). This shows that $F(N)$ should decrease monotonically as a function of $N$ for $N \geq N_{\mathrm{C}}$ and $F(0)$ must be the lowest point of the curve.

In order to address the relative energies of the different film thicknesses quantitatively, we extracted from the time sequence of the STM images in Fig. 1 the fraction of surface area of the different layers as a function of time (Fig. 4). This allowed us to deduce the growth rates (area change per unit time) of the different layers (Table I). Table I shows that, as the local Ag film becomes thicker due to the spontaneous film rearrangement, the mass transfer rate from the void edge to the top of the film becomes slower. We also notice that the overall mass transfer rate is proportional with the void density, but the relative rates between different layers (see Table I) remain unaffected. Here we concentrate on the relative values: During the evolution toward a multilayer film morphology in the presence of voids, the mass transfer always results from the edges of the voids, implying that the same kinetic pathway occurs for the upward motion of the atoms independent of the film thickness. Furthermore, in the time sequence of the multilayer film evolution from 6 to 10 layers shown in Fig. 1, the void density remains essentially constant. Thus, to a good approximation the differences in the rates of mass transfer are directly associated with the differences in the kinetic energy barriers given in Table I. We can therefore

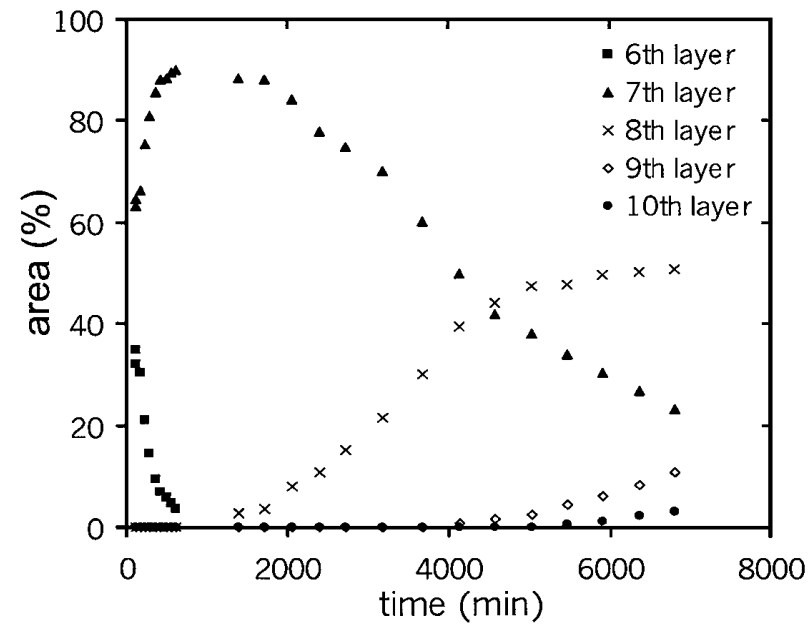

FIG. 4. Time evolution of Ag film morphology acquired from consecutive STM scans after warming up the film to room temperature as shown in Fig. 2. The areas of 6th, 7th, 8th, 9th, and 10th layers are plotted in percentage of surface area.

deduce the relative values of the free energy as a function of the layer thickness, as shown in Fig. 5. The data clearly show a decrease of the free energy with increasing thickness of the Ag film.

Next we determine the absolute energy barrier $B$ separating the phase consisting of a flat (wetting) 6-ML film and the phase consisting of voids and 3D islands (nonwetting equilibrium structure) (see Fig. 3) by assuming a reasonable attempt frequency for the upward motion of the atoms from the voids. Because the atom transfer process could be a collective motion rather than a simple single-atom hopping event, we have used a very wide range of attempt frequencies $\nu$ between $10^{10}-10^{13} \mathrm{~s}^{-1}$, but the estimated kinetic energy barrier still falls within a relatively narrow range of 0.72 to $0.90 \mathrm{eV}$.

From the preceding data we thus determined quantitatively all the key features of the free-energy curve shown schematically in Fig. 3. These values govern the transition from the wetting two-dimensional flat film morphology toward the nonwetting three-dimensional mound structure. We emphasize that our quantitative measurements of the metastability are based on kinetic-thermodynamic principles, and are independent of the mechanism behind the metastability. In the following, we explore the likely driving forces responsible for the free-energy diagram and the corresponding morphological evolution.

TABLE I. Growth rate $r$ of the particular film shown in Fig. 1, ratio of the growth rates for neighboring layers $\left(r_{i} / r_{i+1}\right)$, and energy difference between different layers $E_{i}-E_{i+1}=k T \times$ $\ln \left(r_{i} / r_{i+1}\right)$ for the 7 th to 10 th layers.

\begin{tabular}{cccc}
\hline \hline Layer $i$ & $\begin{array}{c}\text { Growth rate } r \\
\left(\mu \mathrm{m}^{2} / \mathrm{s}\right)\end{array}$ & $\begin{array}{c}\text { Ratio } \\
r_{i} / r_{i+1}\end{array}$ & $\begin{array}{c}E_{i}-E_{i+1} \\
(\mathrm{meV})\end{array}$ \\
\hline 7 & $1.88 \times 10^{-5}$ & 6.80 & 49.84 \\
8 & $2.77 \times 10^{-6}$ & 3.56 & 32.98 \\
9 & $7.78 \times 10^{-7}$ & 2.84 & 27.14 \\
10 & $2.74 \times 10^{-7}$ & & \\
\hline \hline
\end{tabular}




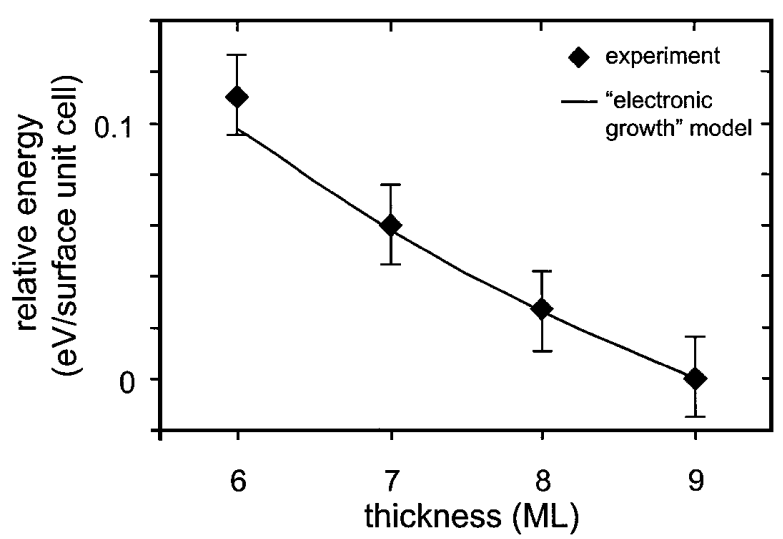

FIG. 5. Experimentally determined relative energy of the 6th, 7th, 8th, and 9th layers (symbols). The theoretical calculations of Ref. [16] is shown as a solid line.

We first note that if the Ag-GaAs system would be governed only by the interface energies, the films would never grow as flat two-dimensional films. Thus, there must be another and more dominant factor capable of modifying the free-energy diagram such that a metastable structure can be reached and stabilized by the above-determined barrier against the wetting-nonwetting transition. As shown convincingly in several recent studies, the motion of conduction electrons in such ultrathin metal overlayers is confined, exhibiting distinct quantum-well states [9-13]. Such quantum nature of the conduction electrons may influence the stability of the overlayers in a dominant way [14-18]. In particular, in the recently proposed "electronic growth" model [16], it is shown that the quantum confinement of the itinerant electrons and the charge transfer between the metal film and the semiconductor substrate can play central roles in the stability of the overlayers, leading to the existence of a critical thickness for smooth growth. When applied to the present system of $\mathrm{Ag} / \mathrm{GaAs}(110)$, this electronic growth model predicts a free energy curve with precisely the same qualitative feature as shown in Fig. 3. In contrast, other mechanisms would have difficulties explaining the observed behavior. For example, elastic strain has been shown to lead to shape transition of islands [19]. However, the strain energy associated with the lattice mismatch can easily be overwhelmed by the quantum size effect in the ultrathin film thickness regime [17]. Furthermore, the strain energy would make the flat film more unstable as the film thickness increases, in contrast to the present observations.

Based on these considerations, we compare our experimental results with the quantitative predictions of the electronic growth model, as shown in Fig. 5. The theoretical values are obtained using the idealized situation where the $\mathrm{Ag}$ film is isolated from the semiconductor substrate by a delta-function potential of infinite strength and the electron effective mass is taken to be 0.5 . Figure 5 shows that very good agreement between theory and experiment is achieved. Within this theory the quantum confinement is the dominating factor above the critical thickness while the charge spilling effect becomes important only at a smaller thickness. The experimentally determined freeenergy curve shows approximately a $1 / \mathrm{N}$ dependence, indicating that the quantum confinement of electrons is mainly responsible for such a behavior [17]. We note that the dominance of the quantum nature of the conduction electrons and the validity of the electronic growth model have also been established in the system of $\mathrm{Pb}$ overlayer growth on $\mathrm{Si}(111)$ [20,21].

In summary, we have studied quantitatively the layerresolved metastability of the flat $\mathrm{Ag}$ thin films grown on $\operatorname{GaAs}(110)$. We deduced the kinetic barrier that the initial system must overcome in order to reach its thermodynamically stable state via the wetting-nonwetting transition. Furthermore, the quantitative results of the free-energy profile obtained from the experiment provide evidence that the metastability of the Ag overlayers is induced predominantly by the confined motion of electrons within the overlayers.

This work was supported by NSF Grants No. DMR0071893, No. CHE-8920120, and No. CTS-9512657, Texas Advanced Research Program, the Welch Foundation, ORNL, managed by UT-Battell, LLC for the U.S. DOE under Contract No. DE-AC05-00OR22725.

[1] A. Pimpinelli and J. Villain, Physics of Crystal Growth (Cambridge University Press, Cambridge, U.K., 1998).

[2] A. R. Smith, K.-J. Chao, Q. Niu, and C. K. Shih, Science 273, 226 (1996).

[3] G. Neuhold et al., Surf. Sci. 376, 1 (1997).

[4] Ph. Ebert, K.-J. Chao, Q. Niu, and C. K. Shih, Phys. Rev. Lett. 83, 3222 (1999).

[5] B. M. Trafas, Y. N. Yang, R. L. Siefert, and J. H. Weaver, Phys. Rev. B 43, 14107 (1991).

[6] N. M. R. Evans, B. Y. Han, and J. H. Weaver, Surf. Sci. 465, 90 (2000).

[7] Hongbin Yu, K.-J. Chao, and C. K. Shih (unpublished).

[8] T. R. Linderoth et al., Phys. Rev. Lett. 77, 87 (1996).

[9] J.E. Ortega and F. J. Himpsel, Phys. Rev. Lett. 69, 844 (1992).

[10] D. A. Evans, M. Alonso, R. Cimino, and K. Horn, Phys. Rev. Lett. 70, 3483 (1993); I. B. Altfeder, K. A. Matveev, and D. M. Chen, Phys. Rev. Lett. 78, 2815 (1997); I. B. Altfeder, D. M. Chen, and K. A. Matveev, Phys. Rev. Lett. 80, 4895 (1998).

[11] J. J. Paggel, T. Miller, and T. C. Chiang, Science 283, 1709 (1999); D.-A. Luh et al., Phys. Rev. Lett. 84, 3410 (2000).

[12] R. K. Kawakami et al., Nature (London) 398, 132 (1999).

[13] Ch. Würsch et al., Nature (London) 389, 937 (1997).

[14] P. J. Feibelman and D. R. Hamann, Phys. Rev. B 29, 6463 (1984).

[15] B. J. Hinch et al., Europhys. Lett. 10, 341 (1989).

[16] Z. Zhang, Q. Niu, and C.-K. Shih, Phys. Rev. Lett. 80, 5381 (1998).

[17] Z. G. Suo and Z. Y. Zhang, Phys. Rev. B 58, 5116 (1998).

[18] D. A. Luh et al., Science 292, 1131 (2001).

[19] J. Tersoff and R. M. Tromp, Phys. Rev. Lett. 70, 2782 (1993).

[20] V. Yeh et al., Phys. Rev. Lett. 85, 5158 (2000).

[21] W. B. Su et al., Phys. Rev. Lett. 86, 5116 (2001). 